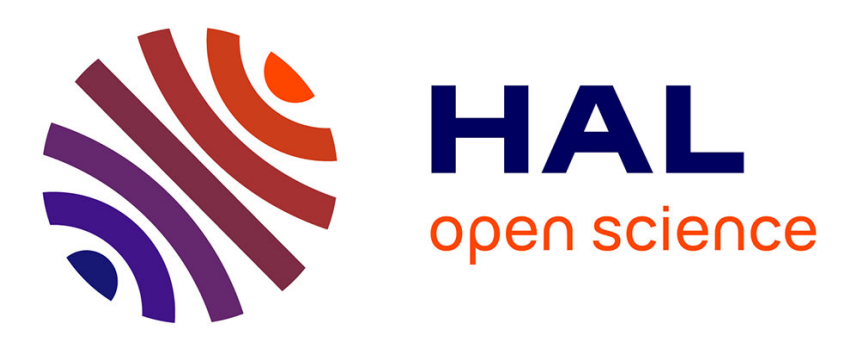

\title{
Assessing the limits of determinism and precision in ultrafast laser ablation
}

M. Garcia-Lechuga, G. Gebrayel El Reaidy, H. Ning, P. Delaporte, D. Grojo

\section{To cite this version:}

M. Garcia-Lechuga, G. Gebrayel El Reaidy, H. Ning, P. Delaporte, D. Grojo. Assessing the limits of determinism and precision in ultrafast laser ablation. Applied Physics Letters, 2020, 117 (17), pp.171604. 10.1063/5.0023294 . hal-02982774

\section{HAL Id: hal-02982774 \\ https://hal.science/hal-02982774}

Submitted on 28 Oct 2020

HAL is a multi-disciplinary open access archive for the deposit and dissemination of scientific research documents, whether they are published or not. The documents may come from teaching and research institutions in France or abroad, or from public or private research centers.
L'archive ouverte pluridisciplinaire HAL, est destinée au dépôt et à la diffusion de documents scientifiques de niveau recherche, publiés ou non, émanant des établissements d'enseignement et de recherche français ou étrangers, des laboratoires publics ou privés. 


\title{
Assessing the limits of determinism and precision in ultrafast laser ablation
}

\author{
M. Garcia-Lechuga, ${ }^{1,2}$ G. Gebrayel El Reaidy, ${ }^{1}$ H. Ning,,${ }^{1}$ P. Delaporte,${ }^{1}$ and D. \\ Grojo ${ }^{1^{*}}$ \\ ${ }^{1}$ Aix Marseille University, CNRS, LP3, UMR7341, 13288 Marseille, France \\ ${ }^{2}$ Departamento de Física Aplicada, Universidad Autónoma de Madrid, Campus de Cantoblanco, 28049 Madrid, Spain \\ * Author to whom correspondence should be addressed. Electronic mail: david.grojo@univ-amu.fr
}

\begin{abstract}
Although ultrafast lasers provide unique capabilities for advanced manufacturing, important challenges remain, since superresolution naturally conflicts with high repeatability in material processing. In this work, we perform statistical analyses of ablation precision at varying pulse durations, down to the sub-picosecond regime. By comparing the results with a 'noise model' accounting for laser fluctuations, we establish that pulses shorter than 200 fs allow us to obtain the highest achievable level of determinism. Our model is a simple extension of an extensively used method proposed by Liu, in which error propagation theory is applied to allow us to derive and reach the limits of precision and reproducibility in laser machining. Its validity is confirmed by an experimental demonstration in which reproducible features as small as $1 / 10$ of the beam size are produced with a stable laser source exhibiting energy fluctuations of $0.3 \%$.
\end{abstract}

As defined in the Cambridge Dictionary, 'precision' is the quality of being exact. In science and engineering, this is commonly associated with both the accuracy of an instrument and the repeatability of a physical process. These aspects have attracted considerable attention in the context of laser fabrication since the significant advances made with the advent of femtosecond lasers for highly accurate material processing ${ }^{1}$. Material modification is limited to the focal volume, an aspect that forms the basis of precise 3D-writing technologies involving transparent dielectrics and semiconductors ${ }^{2,3}$. However, the modification threshold changes from stochastic for long pulses to deterministic for shorter pulses ${ }^{4}$, which makes the interaction process extraordinarily controllable, and allows for super-resolution machining performances 5,6 .

To illustrate this second point, the reader is typically referred to the impressive demonstration given by Joglekar et al. ${ }^{7}$ in which ablation features as small as $40 \mathrm{~nm}$ were produced with strongly focused 800 -fs pulses at a wavelength of $1053 \mathrm{~nm}$. This sub-diffraction limit on machining resolution can be explained by the threshold response of ablation, which is independent of material defects and absorption nonlinearity ${ }^{8}$. This makes it possible to restrict ablation to extremely small areas, far below the laser spot size, provided that the fluence at the center of the laser spot is adjusted to near-threshold conditions. Based on this mechanism, there is in principle no limit on the machining resolution. However, in practice, fluctuations always arise in the space-time characteristics of laser pulses, preventing repeatable digital writing at the 
nanometer scale. The same fluctuations also make it difficult to experimentally evaluate the real level of determinism of the underlying physical mechanisms.

To date, very few experimental studies have attempted to quantify the determinism of the ultrafast laser ablation response, and this is generally addressed by determining the ablation probability as a function of the incident fluence. The most relevant studies ${ }^{9-11}$ using this methodology lead to contradictions, giving rise to an interesting debate. Some argue that the dielectric material damage response is almost perfectly deterministic for picosecond or sub-picosecond pulses ${ }^{9,11}$, while others find thresholds not better defined than about $40 \%$ in relative fluence (transition between zero and $100 \%$ probability of damage) at a pulse duration of $300 \mathrm{fs}$, and a relatively modest improvement for pulse durations as short as 7 fs ${ }^{10}$. This contradiction probably persists because it is difficult to maintain and control all aspects of an experiment while varying a single parameter. In addition, when fluctuating or non-perfectly repeatable features are observed, it is hard to establish whether these originate from stochastic aspects of the interaction physics or from experimental fluctuations. The commonly accepted relationship between a deterministic response and the dominant role of tunneling ionization also raises questions regarding the observations of very reproducible thresholds found for pulse durations of $800 \mathrm{fs}^{7}$, and less reproducible thresholds for pulses of less than $10 \mathrm{fs}{ }^{10}$. While the Keldysh parameter ${ }^{12}$ certainly indicates the important role of tunneling for the shortest pulses, it has minor importance in principle in an ablation experiment with pulses of hundreds of femtoseconds.

In this letter, we focus on clarifying the determinism and the precision limits in ultrafast laser ablation and establishing the connection between them, using pulses at $1030 \mathrm{~nm}$ with durations of $200 \mathrm{fs}$ and 1,2 and 5 ps. Careful metrology of the applied laser conditions and crater characterization by confocal microscopy allow us to compare the results and facilitate discussion. A classical analysis of the ablation probability is performed, and is complemented by a simple but original analysis characterizing the repeatability of laser machining, adapted from Liu's widely used method ${ }^{13}$. These reveal that pulses of $200 \mathrm{fs}$ give a sufficiently high level of determinism to make the laser energy fluctuation the only factor limiting the precision. This allows us to shed light on the remaining challenges in laser technology in terms of delivering a reliable writing solution at the nanometer scale.

The experiments were based on a commercial femtosecond laser (Pharos, Light Conversion) delivering linearly polarized pulses at a wavelength of $1030 \mathrm{~nm}$. The compressor of the laser system was motorized and calibrated so that the investigations were done at a pulse duration of $200 \mathrm{fs}$ and repeated with chirped pulses of 1,2 and 5 ps while maintaining the 
same pulse-to-pulse energy stability of about $0.3 \%$ (standard deviation, SD) as shown in Fig. 1 (a). The spectral and spatial stability characteristics of the beam were also verified, as any source of fluctuation could have affected the experiments ${ }^{14}$. The measurement of an excellent spectral stability reasonably translates into good temporal stability for the chirped pulses (see the Supplementary section for details of the metrology).

The irradiation setup contained a filtering aperture $(4 \mathrm{~mm})$ before a focusing lens of focal length $50 \mathrm{~mm}$, a sample holder on XYZ motorized stages, and an in situ microscope for precise sample positioning, as used in Ref. ${ }^{8}$. Pulse energy control using a combination of a half-wave plate and polarizer allowed us to perform experiments at different energies, which were characterized using a pyroelectric energy meter (PE9-C, Ophir). A total of 16 irradiations at normal incidence were repeated for each energy value, distributed in a 4 x 4 matrix with separation of $50 \mu \mathrm{m}$. The sample was a sapphire window of thickness $1 \mathrm{~mm}$ and c-cut orientation. The choice of this target material was motivated by the very neat craters produced with ultrashort pulses, without apparent thermally affected zones ${ }^{8}$ that can introduce ambiguity. Characterization of the ablated surface was performed by means of confocal microscopy (Leica DCM3D, $460 \mathrm{~nm}$ illumination, 150x objective lens). This methodology has an advantage over other microscopy techniques in that surfaces can be characterized based on their topography rather than on changes in contrast, which may depend on the type of modifications achieved in different interaction regimes ${ }^{15}$. The use of topographic images together with automated software analyses (Mountains 8) allowed us to monitor the onset of ablation and properly determine the ablated area. In all the analyses, the same criterion for ablation was applied (removal of material to a depth of $10 \mathrm{~nm}$ ), independent of the pulse duration and the shape of the induced modification. This criterion avoided any visual interpretation and solved the issue of subjectivity, as it did not depend on the observer.

Fig. 1 (b) shows the measurements of the ablated area induced in sapphire after single-shot irradiation at different pulse energies, for pulse durations of $200 \mathrm{fs}$ and 1,2 and $5 \mathrm{ps}$. The mean values of the ablated areas over 16 irradiations are shown with symbols. Using this format, the graph displays all energy conditions for which ablation is detected, even if it only occurs on a single irradiated site (with non-zero probability). In addition, vertical bars indicate the span between the maximum and minimum sizes of the ablated crater for each statistical analysis, allowing for direct observation of the degradation in repeatability with increased pulse durations in the picosecond range. For a more visual assessment, Fig. 1(c-d) shows the images corresponding to the maximum and minimum ablated areas for each pulse duration, in a series of 16 irradiations at energies leading to similar crater sizes for the different pulse durations. At $200 \mathrm{fs}$, almost perfect replicas are observed for both energy levels tested, while differences in size and morphology are clearly visible for pulses of 1 ps at near-threshold 
conditions, and under all tested experimental conditions at 2 ps. These observations are taken as initial evidence of a reduction of determinism in the picosecond domain.
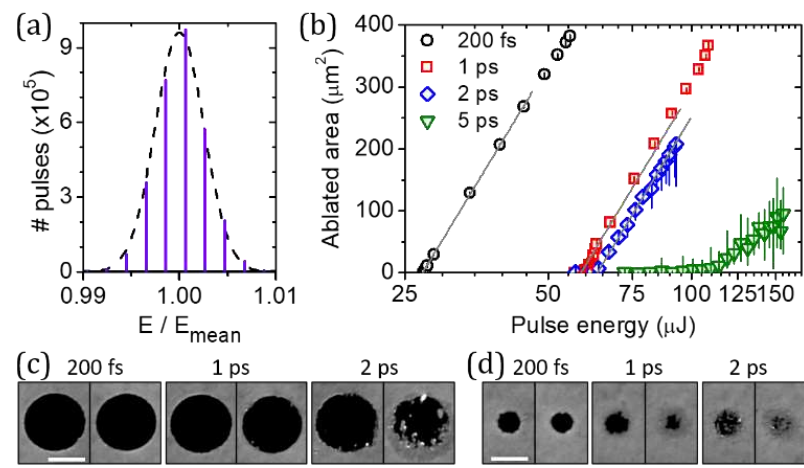

(d) $200 \mathrm{fs}$

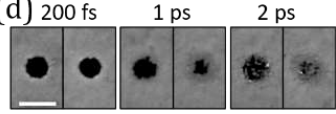

Fig. 1. (a) Measured pulse energy distribution over 300,000 consecutive pulses and the corresponding Gaussian noise function (dashed line); (b) ablated areas on sapphire produced after irradiation with single pulses $(\lambda=1030 \mathrm{~nm})$ of different durations. Symbols: mean ablated area over 16 craters produced at a fixed energy. Vertical bars: span between minimum and maximum crater sizes at a fixed energy. Grey line: linear fitting (Eq.1) for a Gaussian beam waist of $18.7 \mu \mathrm{m}$. (c-d) Confocal microscopy images of the surface modifications. Black regions correspond to depressions below a depth of $10 \mathrm{~nm}$. The selected images correspond to the minimum and maximum ablated areas over a set of 16 irradiated sites (scale bar: $10 \mu \mathrm{m}$ ). In (c), craters are produced at $\sim 1.5$ times the energy threshold for ablation for each case. In (d), craters are produced at $\sim 1.05$ times the threshold.

The data in Fig. 1(b) are also represented according to Liu's 'thresholding' method ${ }^{13}$, which allows to retrieve under a Gaussian spot approximation the beam waist (radius at $1 / \mathrm{e} 2$ ), $w_{0}$, and the energy threshold of ablation, $E_{t h}$. This gives rise to a linear mathematical relationship between the ablated area, $A$, and the logarithm of the pulse energy, $\ln (E)$, as follows:

$$
\mathbf{A}(\mathbf{E})=\frac{\pi \mathbf{w}_{0}^{2}}{2} \ln \left(\mathbf{E} / \mathbf{E}_{\mathrm{th}}\right)
$$

Applying this linear fit to the ablated areas induced with pulses of $200 \mathrm{fs}$, we obtain $w_{0}=18.7 \mu \mathrm{m}$ and $E_{t h}=27.3 \mu \mathrm{J}$. From the relationship that relates the fluence and the energy for a Gaussian beam, $F_{t h}=2 \cdot E_{t h} /\left(\pi \cdot w_{0}^{2}\right)$, we obtain $F_{t h}=$ $5.0 \mathrm{~J} / \mathrm{cm}^{2}$. Using the same waist for the measurements at $1 \mathrm{ps}$ and $2 \mathrm{ps}$, we obtain $F_{t h}=10.6 \mathrm{~J} / \mathrm{cm}^{2}$ and $11.6 \mathrm{~J} / \mathrm{cm}^{2}$. The material responses at $5 \mathrm{ps}$ tend to make this fitting procedure invalid, and the threshold is derived below.

To further explore the determinism with ultrashort pulses, we represent the ablation probability as a function of the pulse energy, as in Refs. ${ }^{9-11}$. The data are plotted in Fig. 2, where the x-axis is normalized with respect to the ablation energy threshold to enable direct comparisons between different pulse durations. The ablation energy threshold is arbitrarily taken as 
the energy associated with a $50 \%$ probability of ablation (half transition). The energy values obtained using this definition and those obtained with Liu's method differ by less than $2 \%$ for pulses of $200 \mathrm{fs}$ and 1 ps. However, the value obtained at 2 ps is about $7 \%$ lower than that derived from Liu's method. For a pulse duration of 5 ps, we obtain $E_{t h}=87 \mu \mathrm{J}$, which corresponds to $F_{t h}=16 \mathrm{~J} / \mathrm{cm}^{2}$. In order to account for the limited size of our statistical sample (N=16 irradiations), we include a vertical error bar representing the statistical error $(\mathrm{SE})$ in the probability $(\mathrm{P})$ determination $S E(P)=$ $\sqrt{(P \cdot(1-P) / N)}$

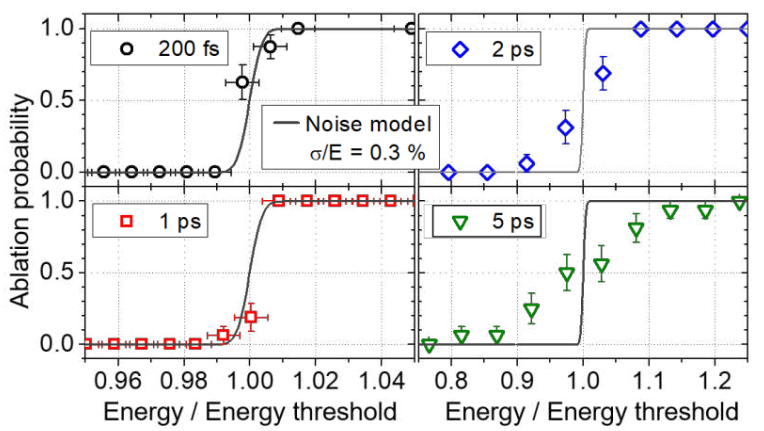

Fig. 2. Laser ablation probability as function of pulse energy (normalized to threshold) for different pulse durations. The horizontal error bars represent the pulse-to-pulse energy fluctuations, while the vertical bars indicate an estimate of the minimum statistical error due to sampling. Solid lines show a comparison with a 'noise model' that accounts only for the measured energy fluctuations (see Eq. 3).

A sharp transition in the probability of ablation $\left(\cong \pm 1 \% \cdot E_{t h}\right)$ is observed in Fig. 2 for pulse durations of 200 fs and 1 ps. The transition becomes significantly broader for pulses of 2 and 5 ps $\left( \pm 10 \% \cdot E_{t h}\right.$ and $\left.\cong \pm 20 \% \cdot E_{t h}\right)$, providing further direct evidence of a lack of determinism for these pulse durations. Furthermore, our observation is consistent with those of previous works ${ }^{4,9}$, which associated the lack of determinism with the increasing importance of avalanche ionization and an associated sensitivity to precursor defects in the picosecond domain. However, the Keldysh parameter of $\gamma \approx 1.8$ for the intensities used for the $200 \mathrm{fs}$ pulse tends to contradict the essential role played by tunneling ionization in obtaining the deterministic material ablation response, as suggested by other authors ${ }^{7,10}$.

To attribute the sharp transition observed in Fig. 2 for pulses of $200 \mathrm{fs}$ and 1 ps to a deterministic laser/matter interaction, it is necessary to deconvolute the experimental fluctuations. We therefore present comparisons with predictions that account for the measured energy fluctuations (Fig. 1 (a)), which can be fitted to a Gaussian noise function as follows: 


$$
\rho(E, \bar{E})=\frac{1}{\sigma(\bar{E}) \sqrt{2 \pi}} e^{\left(-\frac{1(E-\bar{E})^{2}}{2 \sigma^{2}(\bar{E})}\right)}
$$

with a ratio between the SD, $\sigma(\overline{\boldsymbol{E}})$, and the mean value of the distribution, $\overline{\boldsymbol{E}}$, of $0.3 \%$. Accounting only for these energy fluctuations, we apply a 'noise model' of the probability of ablation, based on integrals of the density function:

$$
P(\bar{E})=\int_{E_{t h}}^{\infty} \rho(E, \bar{E}) d E
$$

By applying this equation for different values of $\overline{\boldsymbol{E}}$ that are above and below $E_{t h}$, we can calculate the ablation probability as a function of the pulse energy, as shown by the solid lines in Fig. 2 with the experimental data. An initial conclusion is that this simple 'noise model' compares relatively well with the experimental data at $200 \mathrm{fs}$ and $1 \mathrm{ps,} \mathrm{unlike} \mathrm{the}$ data for longer pulses. This can be attributed to a high deterministic response. The modest discrepancies observed for $200 \mathrm{fs}$ and 1 ps can be reasonably attributed to the size of the statistical sample (horizontal error bars), which is insufficient to ensure a well-established Gaussian noise distribution.

In addition, since determinism is inherently associated with precision and repeatability, a statistical analysis of crater sizes is also instructive. In Fig. 3 (a), we show the fluctuation in the ablated area, $\sigma_{A} / \bar{A}$ (where $\bar{A}$ is the mean value and $\sigma_{A}$ is the SD over the 16 ablated areas), as a function of the excitation level $\left(E / E_{t h}\right.$ or $\left.F / F_{t h}\right)$. This alternative representation shows the decrease in the reproducibility when the pulse duration is increased. It is interesting to note that it shows a difference between $200 \mathrm{fs}$ and 1 ps, which was more difficult to obtain with the other analyses. At 200 fs, we note the excellent stability of the features, with a fluctuation in their size of less than $1 \%$ at a fluence significantly exceeding the ablation threshold. We also note that a behavior common to all the measurements is an increase in the fluctuation at nearthreshold conditions. To describe this observation, we use a derivation of Liu's method to account for the laser energy fluctuations, as proposed in Ref. ${ }^{16}$. By applying the error propagation theory in Eq. 1, we obtain an expression for the ablated area fluctuations,

$$
\frac{\sigma_{A}}{A}=\left(\ln \left(\frac{\mathrm{E}}{E_{t h}}\right)\right)^{-1} \cdot \frac{\sigma_{E}}{E}
$$

The results of this calculation for laser fluctuations of $\sigma / E=0.3 \%$ are plotted with a solid line in Fig. 3 (a). The curve describes the increase in the size fluctuations as the peak fluence approaches the ablation threshold, and exhibits reasonable agreement with experiment at a pulse duration of $200 \mathrm{fs}$. At this stage, it should be noted that it would not be correct to 
conclude that the results obtained at pulse duration of $200 \mathrm{fs}$ show perfect, physically deterministic behavior. However, the excellent energy stability of $0.3 \%$ (SD) for this experiment directly indicates a lower limit for the high level of determinism under these conditions. From a fundamental perspective, we cannot definitively conclude that 200 fs gives a perfectly deterministic response, since this requires us in principle to deconvolute all potential sources of noise in the experiment, while our model accounts only for laser energy fluctuations. Although we can confirm the presence of extremely low laser fluctuations in the temporal, spectral and spatial domains (see the Supplementary Material section), this analysis shows that the physical response fluctuations (if any) are apparently so low that a proper deconvolution may require a more advanced methodology to account for additional sources of low levels of noise in the experimental conditions.

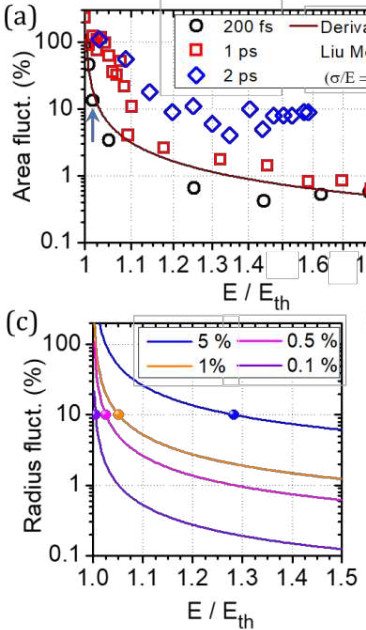

(b) $\mathrm{E}=1.02 \cdot \mathrm{E}_{\mathrm{th}}$

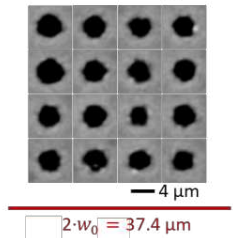

Fig. 3. (a) (Symbols) Ablated area fluctuations based on statistical analyses of 16 irradiation sites at each laser condition. (Line) Calculation derived from Liu's method that accounts for the measured laser energy fluctuations (Eq. 4). (b) Craters produced at nearthreshold conditions (see arrow in (a)) with pulses of $200 \mathrm{fs}$. (c) (Lines) Laser machining repeatability calculations for laser stabilities of between $0.1 \%$ and $5 \%$ (Eq. 6). (Symbols) Lower energy limit for obtaining craters with fluctuations of below 10\%. (d) (Line) Crater size as a function of the excitation energy level (Eq. 1). (Symbols) Minimum crater size with fluctuations of below $10 \%$ that is achievable with the laser stabilities shown in (c).

However, on the technological front, we can be more definite about the significance of our observations. Firstly, we establish that a pulse duration of $200 \mathrm{fs}$ is short enough to give highly reproducible regimes. Even if we hypothesize that some stochastic responses remain at this pulse duration, their influences are negligible in comparison to the inherent laser fluctuations. Secondly, we demonstrate that the assumptions of Liu's method, which is widely used in machining studies, allow us to predict not only the size (Eq. 1) but also the repeatability of the feature (Eq. 4). This observation is important 
because it allows us to quantify the trade-off between repeatability and resolution, two aspects that are essential in defining the precision of a technology, as shown in Figs. 3 (c) and (d), respectively. As can be seen from Fig. 3 (d), there is in principle no physical limitation on the resolution that is achievable at near-threshold peak fluences (Eq. 1). However, Fig. 3(c) shows an asymptotic behavior of the fluctuations that tends to infinity at rates correlated with the stability of the laser. To illustrate the implications of this latter aspect, the circular symbols in Fig. 3(d) show the minimum feature size that is obtainable with a variation tolerance of $10 \%$, calculated for the different laser stabilities in Fig (c). It can be seen that craters smaller than $w_{0} / 3$ can be routinely produced, even with modest energy stability. A much higher precision of $r=w_{0} / 10$ is expected from exploiting the stability of our laser $(0.3 \% \mathrm{SD})$, as shown by the experimental results in Fig 3 (b).

Although we have focused on sapphire here, we have also tested other dielectrics such as fused silica and soda lime glass in order to confirm that the general 'precision abacus' derived in Fig. 3 remains valid for these materials and can serve as a general guideline for technology development. However, we anticipate that our simple hypotheses, which are based on a pure local fluence response, will not rigorously hold when extreme precision is achieved, due to the increasing importance of energy diffusion and dissipation phenomena at the nanoscale ${ }^{17}$ and/or when the surrounding affected zones become limiting aspects ${ }^{8}$. In support of our conclusions, it is also interesting to note that our analysis allow to make a comprehensive review of the literature. In the pioneering work of Joglekar et al. ${ }^{7}$, repeatable arrays of holes with diameter $350 \mathrm{~nm}$ were demonstrated. This situation corresponds to $r \cong w_{0} / 3$, where the value of $w_{0} \approx 550 \mathrm{~nm}$ is inferred from the Abbe limit for $\mathrm{NA}=1.3$ and $\lambda=1053 \mathrm{~nm}$. Our analysis shows that this resolution can be achieved even with a modest laser energy stability (4\%). However, the $40 \mathrm{~nm}$ diameter hole ${ }^{7}$ is inevitably non-repeatable, since this resolution level $\left(w_{0} / 30\right)$ cannot be maintained without the use of a super-stable laser $(<<0.1 \%)$

In summary, a strict threshold nature of the femtosecond laser ablation of dielectrics is observed at a pulse duration of $200 \mathrm{fs}$ One consequence is the possibility of obtaining super-resolution machining by slightly exceeding the fluence ablation threshold, without the need for more sophisticated laser technologies for shorter pulses. As demonstrated by our experimentally validated 'noise' model, however, the precision becomes limited by the laser stability using this strategy. Our statistical treatment clearly demonstrates why highly reproducible laser processing with sub-100 nm precision remains an important problem. It therefore offers both a comprehensive vision of the current limitations and tools that will be crucial in inspiring practical solutions to this exciting precision problem.

Supplementary material. See the supplementary material for an additional analysis of laser fluctuations. 
Funding. H2020 European Research Council (724480).

Acknowledgments. We thank O. Utéza and N. Sanner (LP3) for fruitful discussions.

Data availability: The data supporting the findings of this study are available from the corresponding author upon request.

\section{References}

${ }^{1}$ M. Malinauskas, A. Žukauskas, S. Hasegawa, Y. Hayasaki, V. Mizeikis, R. Buividas, and S. Juodkazis, Light Sci. Appl. 5, e16133 (2016)

${ }^{2}$ C.B. Schaffer, A. Brodeur, and E. Mazur, Meas. Sci. Technol. 12, 1784 (2001).

${ }^{3}$ M. Chanal, V.Y. Fedorov, M. Chambonneau, R. Clady, S. Tzortzakis, and D. Grojo, Nat. Commun. 8, 773 (2017).

${ }^{4}$ B.C. Stuart, M.D. Feit, S. Herman, A.M. Rubenchik, B.W. Shore, and M.D. Perry, J. Opt. Soc. Am. B 13, 459 (1996).

${ }^{5}$ F. Korte, J. Serbin, J. Koch, A. Egbert, C. Fallnich, A. Ostendorf, and B.N. Chichkov, Appl. Phys. A Mater. Sci. Process. 77, $229(2003)$

${ }^{6}$ Z.Z. Li, L. Wang, H. Fan, Y.H. Yu, H.B. Sun, S. Juodkazis, and Q.D. Chen, Light Sci. Appl. 9, 41 (2020).

${ }^{7}$ A.P. Joglekar, H. -h. Liu, E. Meyhofer, G. Mourou, and A.J. Hunt, Proc. Natl. Acad. Sci. 101, 5856 (2004).

${ }^{8}$ M. Garcia-Lechuga, O. Utéza, N. Sanner, and D. Grojo, Opt. Lett. 45, 952 (2020).

${ }^{9}$ D. Du, X. Liu, G. Korn, J. Squier, and G. Mourou, Appl. Phys. Lett. 64, 3071 (1994).

${ }^{10}$ N. Sanner, O. Utéza, B. Chimier, M. Sentis, P. Lassonde, F. Légaré, and J.C. Kieffer, Appl. Phys. Lett. 96, 3 (2010).

${ }^{11}$ B. Mangote, L. Gallais, M. Zerrad, F. Lemarchand, L.H. Gao, M. Commandré, and M. Lequime, Rev. Sci. Instrum. 83, 013109 (2012).

${ }^{12}$ L.V. Keldysh, Sov. Phys. JETP 20, 1307 (1965).

${ }^{13}$ J.M. Liu, Opt. Lett. 7, 196 (1982).

${ }^{14}$ A. Ollé, J. Luce, N. Roquin, C. Rouyer, M. Sozet, L. Gallais, and L. Lamaignère, Rev. Sci. Instrum. 90, (2019).

${ }^{15}$ M. Garcia-Lechuga, J. Solis, and J. Siegel, Appl. Phys. Lett. 108, 171901 (2016). 
${ }^{16}$ J. Mur and R. Petkovšek, Appl. Phys. A 124, 62 (2018).

${ }^{17}$ A. Gil-Villalba, C. Xie, R. Salut, L. Furfaro, R. Giust, M. Jacquot, P.A. Lacourt, J.M. Dudley, and F. Courvoisier, Appl. Phys. Lett. 107, 061103 (2015). 


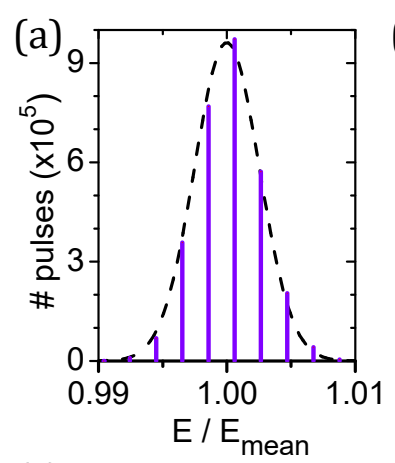

(c) $200 \mathrm{fs}$
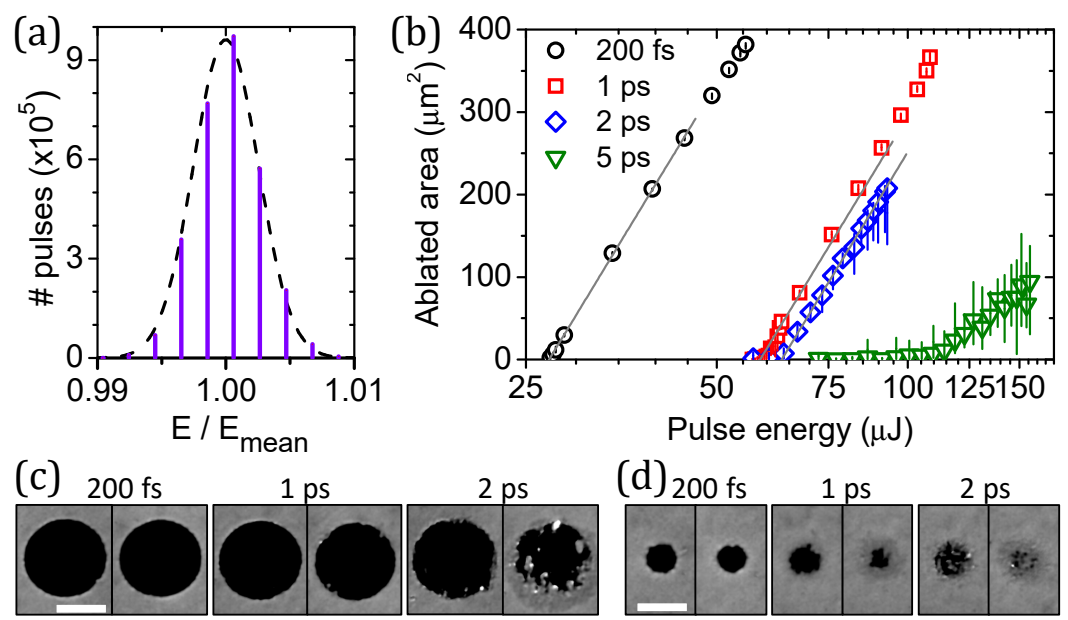

(d)

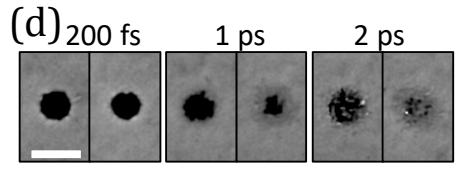




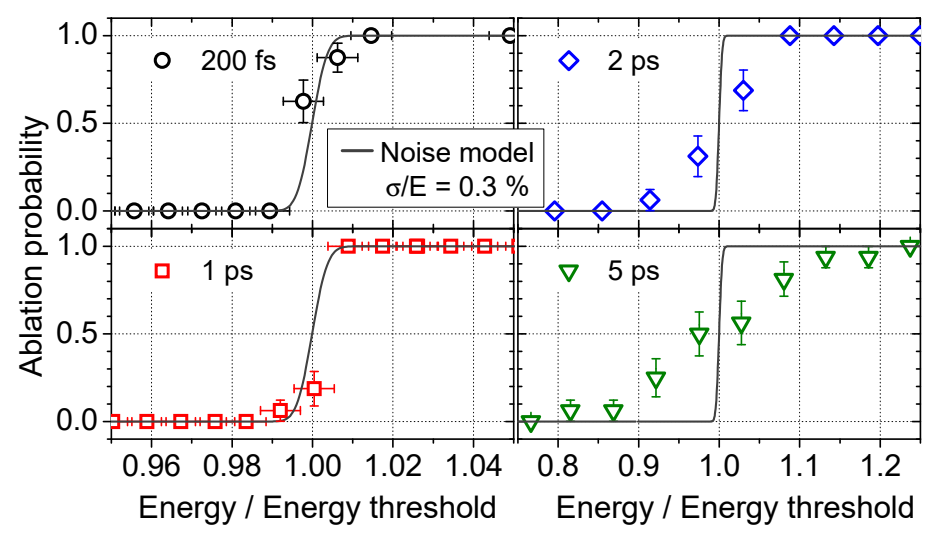



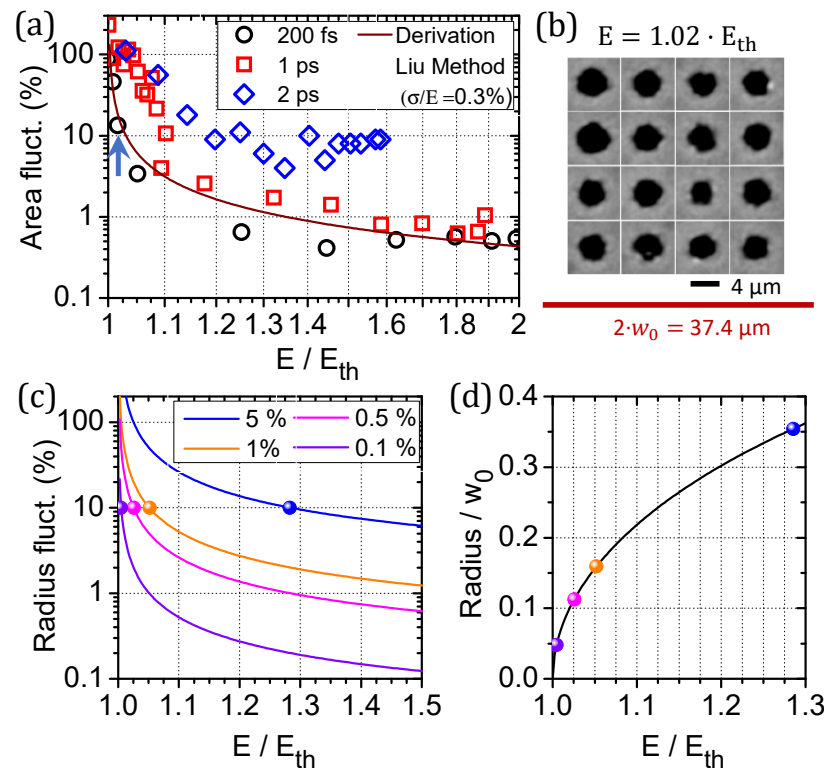